Itinéraires Itinéraires

Littérature, textes, cultures

\title{
« Regarder le corps » dans la mythologie japonaise : tabou ou vertu?
}

Ignacio Quiros

\section{OpenEdition}

Journals

Édition électronique

URL : http://journals.openedition.org/itineraires/1490

DOI : $10.4000 /$ itineraires. 1490

ISSN : 2427-920X

Éditeur

Pléiade

\section{Édition imprimée}

Date de publication : 1 novembre 2011

Pagination : 33-44

ISBN : 978-2-296-55720-8

ISSN : $2100-1340$

\section{Référence électronique}

Ignacio Quiros, « « Regarder le corps » dans la mythologie japonaise : tabou ou vertu ? », Itinéraires [En ligne], 2011-3 | 2011, mis en ligne le 01 novembre 2011, consulté le 01 mai 2019. URL : http:// journals.openedition.org/itineraires/1490; DOI : 10.4000/itineraires.1490

\section{(ब) $\odot \Theta$}

Itinéraires est mis à disposition selon les termes de la licence Creative Commons Attribution - Pas d'Utilisation Commerciale - Pas de Modification 4.0 International. 


\title{
« Regarder le corps » dans la mythologie japonaise : tabou ou vertu?
}

\begin{abstract}
In the Kojiki and the Nihon-shoki, mythological records of ancient Japan, we can find many references to nudity. Naked body is often exposed to the eye, and depending on the episode, this association nudity-look is at the root of a wide range of reactions, from fascination to shame, from laughter to submission. Sometimes taboo, sometimes virtue, this relationship between nudity and look needs to be clarified, in order to better understand the body's conception particular to these texts and to ancient Japanese culture.
\end{abstract}

Keywords : nudity, look, taboo, Kojiki, Nihon-shoki

Mots clés : nudité, regard, tabou, Kojiki, Nihon-shoki

Quel rapport les Japonais de l'époque archaïque pouvaient-ils bien entretenir avec leur corps? Voici une question qui, bien qu'épineuse de par le peu de données et de textes existants, mérite bien d'être traitée. Étant donné que les croyances et modes de pensée continentaux n'étaient alors que récemment arrivés et donc très peu présents sur l'archipel, il est légitime de penser que l'on puisse extraire, à travers les textes conservés de cette époque, des fragments épars d'une conscience autochtone du corps.

Le Kojiki et le Nihon-shoki, anciens récits mythologiques et pseudo-historiques, sont les textes les plus anciens du Japon et à ce titre peuvent être considérés comme les plus susceptibles de témoigner de cette conscience archaïque du corps. Effectivement, un simple survol des épisodes mythologiques de ces textes nous apprendra rapidement que les références au corps sont nombreuses : détails particulièrement « crus » tels que démembrements, corps trucidés, déjections, mais également des références aux organes génitaux, domaine qui possède un relief supplémentaire, car la nudité se présente intimement associée au regard extérieur, que ce soit du conjoint ou d'un éventuel « public».

Selon les circonstances du récit, ce regard porté sur le corps est à la base d'un éventail de réactions très variées, allant de la fascination à 
la honte, du rire à la soumission. De plus, loin de s'arrêter à de simples expressions du sentiment, ces réactions ont en général des conséquences dramatiques dans le récit, comme une rupture à jamais entre deux conjoints et par la suite la création d'un clivage éternel entre deux mondes mythiques. C'est le cas des deux démiurges Izanaki et Izanami, long épisode que nous avons baptisé « de l'attirance à la honte » et qui se trouve d'ailleurs au point de départ de notre recherche. La fin tragique de ce récit nous fait penser à l'existence d'un certain tabou par rapport au corps nu et à son exposition au regard extérieur.

Or, ce regard sur le corps peut avoir aussi des conséquences positives. L'exemple le plus représentatif est celui de la déesse Ame no Uzume et sa célèbre danse nue devant la grotte d'Iwato, où la déesse du soleil s'était cachée. Ce spectacle rituel devant le regard des huit myriades de dieux deviendra le déclencheur de la sortie d'Amaterasu et par la suite de la réapparition de la lumière dans le monde.

Il s'agira donc ici de clarifier les rapports entre le corps, le regard et son interprétation dans la culture japonaise archaïque. Certes, nos deux objets de recherche, le Kojiki et le Nihon-shoki, se caractérisent par un langage symbolique assez particulier qui rendra notre tâche quelque peu ardue du fait du grand nombre d'interprétations possibles, mais nous allons malgré tout essayer de construire une grille de lecture archaïque du " corps regardé ».

\section{Le corps, le péché et la honte}

S'il est déjà difficile d'essayer de reconstruire la pensée ancienne d'une civilisation, cela l'est d'autant plus quand on rentre dans le domaine de la conscience du corps. Car, comme le disait Francis Prost, le corps n'est pas un objet historique en tant que tel : on part toujours à la conquête de cet objet à partir de sa propre expérience - celle de son propre corps -, qu'on ne parvient pourtant jamais à saisir comme une extériorité : le fonctionnement corporel nous échappe. En tout cas il n'est jamais entièrement maîtrisé. Si l'on veut saisir le corps d'autrui, a fortiori le corps de personnes disparues depuis plus ou moins longtemps, ce corps-là reste toujours un étranger, qu'on ne peut jamais faire sien : comment restituer les expériences du corps antique, médiéval ou moderne et en expliquer les transformations si l'on est incapable de s'approprier ne serait-ce qu'un peu des sentiments, des passions, des manières de penser ou d'agir qui forment le corps d'autrui? Ce paradoxe du corps tellement confondu et mêlé (Descartes), l'historien en mesure toutes les conséquences dans son propre champ : « le corps n'est en fait jamais un objet historique en tant que tel, mais est toujours disséminé dans des tissus de relations qui le dépassent ou le sous-tendent ${ }^{1}[\ldots] »$.

1. Francis Prost, «Introduction », dans Francis Prost et Jérôme Wilgaux (dir.), Penser et représenter le corps dans l'Antiquité, Actes du colloque international de Rennes (1-4 septembre 2004), Rennes, PUR, 2006, p. 8. 
Cette difficulté de saisir tout le relief de la pensée ancienne augmente encore lorsqu'il s'agit d'une civilisation dont le système de pensée est très éloigné de celui du chercheur. C'est bien pour cela que les divers contacts de l'Occident avec le Japon depuis le XVI ${ }^{\mathrm{e}}$ siècle nous ont livré au long de l'histoire des interprétations très diverses sur la façon dont les Japonais d'autrefois " pensaient leur corps ». D'une manière générale, ces interprétations sont basées sur les expériences personnelles des auteurs et se bornent à l'environnement et aux personnages qu'ils ont côtoyés. Voilà pourquoi il faut prendre avec précaution les conclusions générales qu'ils avancent, ces dernières négligeant souvent des facteurs comme la diversité régionale, les classes sociales, le passé historique ou la différence entre le monde laïque et religieux.

Or, parmi ces commentaires stéréotypés il est possible de trouver parfois des observations intéressantes, comme celle du missionnaire jésuite espagnol Alejandro Valignano, arrivé au Japon à la fin du XvI siècle. Dans son Sommaire des choses du Japon (1583), il avance qu'au Japon il n'y a pas de conscience du péché ${ }^{2}$. Il faisait référence notamment aux péchés en rapport avec le corps, autrement dit aux péchés charnels.

Cette observation est en rapport avec les affirmations de Ruth Benedict, qui oppose les « cultures du péché » aux « cultures de la honte », en donnant l'Occident comme exemple représentatif des premières, et le Japon comme exemple représentatif des secondes ${ }^{3}$. Comme nous le rappelle l'anthropologue japonaise Yamada $\mathrm{Naomi}^{4}$, les observations de Benedict sont assez naïves en ce sens qu'elles simplifient beaucoup de facteurs comme ceux que l'on a mentionnés ci-dessus, bien qu'elles puissent servir d'outil provisoire pour une étude ethnologique. Yamada affirme que le rapport général que Benedict établit entre la culture japonaise et la honte est notamment valable dans le cas du Japon archaïque ${ }^{5}$.

Cette conscience de la honte dans le Japon archaïque peut être retrouvée dans les récits mythologiques qui feront l'objet de notre étude, le Kojiki et le Nihon-shoki. Ce qui est intéressant est qu'elle s'y trouve intimement reliée au corps nu. La nudité provoque un sentiment de honte dans certains épisodes, mais il est important de souligner, même si cela peut paraître superflu, la présence incontournable d'un déclencheur : le regard. La honte dans ces récits est souvent véhiculée par le regard du partenaire porté sur le corps de l'autre.

2. Alejandro Valignano, Sumario de las cosas de Japón (1583), Tôkyô, Sophia University, 1954.

3. Ruth Benedict, The Chrysanthemum and the Sword: Patterns of Japanese Culture, Boston, Houghton Miffin, 1946, p. 222-224.

4. Yamada Naomi, « Kihantoshite no haji : kiki ni miru kinki to haji no taiô » [La honte en tant que norme : les parallélismes décelables entre la honte et le tabou dans le Kojiki et le Nihonshoki], Kokubungaku nôto, 1990, p. 1.

5. Ibid., p. 2. 


\section{Le tabou du regard}

Pour donner une définition quelque peu simpliste qui s'inscrirait dans le cadre de notre étude, nous considérons que la honte est un sentiment éveillé par le regard de l'autre sur soi. C'est souvent la conscience d'être regardé qui est en cause, et ce « poids du regard » est facilement compréhensible car tout le monde a vécu des situations embarrassantes où l'on préfèrerait «mourir que de continuer à être regardé ».

Cette expression figurée du « poids du regard » est d'autant plus valable dans le cas du Japon archaïque car le regard était censé, selon Nakanishi Susumu ${ }^{6}$, posséder un pouvoir magique à cette époque.

Nous observons ainsi, dans les principaux récits de cette époque, que la relation de personnages ayant ressentis de la honte à cause du regard de l'autre se solde non seulement par une simple dispute mutuelle ou une rupture totale, mais aussi par une succession de conséquences meurtrières, comme l'instauration de la mort dans le monde ou la séparation à jamais de deux mondes mythiques.

Ces conséquences démesurées nous font penser à l'existence d'un tabou, ce qui est facilement vérifiable dans ces textes car l'expression « ne me regarde pas " y apparaît souvent, notamment dans le cas des couples. S'il est déjà humain de vouloir regarder quand on nous a interdit de le faire, ce désir est encore plus présent dans les textes japonais dont il est question ici. Effectivement, les cas du mari voyeur sont nombreux, et cette expression «ne me regarde pas » est reliée directement au corps. C'est donc le regard sur le corps du partenaire qui est tabou, mais il est nécessaire de préciser cette affirmation car les épisodes qui comportent ce phénomène sont contextuellement très différents.

Nous sommes loin d'être des pionniers en ce qui concerne le tabou du regard porté sur le corps dans les récits mythiques anciens japonais. Matsumura $\mathrm{Takeo}^{7}$ avait déjà développé cette thématique, mais comme il nous a semblé qu'il fallait encore préciser certains points, nous allons réétudier ce sujet sous une autre lumière. En effet, les thèses écrites sur le corps et le regard se limitent généralement aux épisodes où il s'agit de tabou.

Dans un premier temps, nous nous efforcerons d'apporter une vision différente de la notion de tabou dans ces épisodes mais le caractère novateur de notre recherche, dans un second temps, portera sur l'ambivalence de ce sujet, en étudiant d'autres exemples où le regard porté sur le corps, loin d'être une action interdite, se relie à des réactions positives telles que l'attirance, le rire ou le respect.

6. Nakanishi Susumu, Kojiki wo yоти [Lire le Kojiki], Shikisha, 1986.

7. Matsumura Takeo, Nihonshinwa no kenkyû [Recherche sur les mythes japonais], Iwanami shoten, 1958, p. 559-584. 


\section{Izanaki et Izanami}

\section{De l'attirance...}

Dans nos mythes, le regard sur le corps nu et l'attirance se trouvent rarement associés. Comme nous le verrons plus tard, les exemples où la vue de la nudité se relie à la honte sont beaucoup plus nombreux. Il existe cependant un épisode où ces sentiments par rapport à la nudité ne sont pas exclusifs, mais où attirance et honte sont bien présents, l'attirance se muant en honte au fur et à mesure que progresse le récit. Il s'agit du cas des « parents du monde », Izanaki et Izanami, le couple de dieux qui a créé la Terre, le Japon en l'occurrence, en plongeant une hallebarde dans les eaux de la mer première. Des gouttes d'eau salée tombant de l'hallebarde s'est formée une île, Onogoro, où ils sont descendus et ont exécuté un rituel de procréation en comparant d'abord leurs corps et puis en tournant autour du pilier céleste ${ }^{8}$.

À la différence des premiers dieux de la généalogie liminaire qui n'avaient point de corps, Izanaki et Izanami ont bien un corps anthropomorphe, les épisodes d'enfantement des îles et de la deuxième génération de dieux nous le prouvent. Mais qu'en est-il de leurs vêtements? Il pourrait être tentant d'imaginer nos deux démiurges, Izanaki et Izanami, à l'image d'Adam et Ève, nus dans l'île paradisiaque d'Onogoro qu'ils viennent eux-mêmes de créer, là où la honte de la nudité n'existe pas. Mais, il s'agirait là d'une supposition gratuite qui ne tiendrait pas compte de la complexité de la réalité.

Certes, le texte ne nous donne aucune indication explicite par rapport à la nudité des dieux. En général, dans les épisodes ultérieurs, beaucoup de dieux sont revêtus de tuniques ou d'habits guerriers, ce qui nous autorise à supposer que les divinités sont en principe vêtues. Si c'est aussi le cas pour Izanaki et Izanami, à l'instar de ces celèbres représentations un peu trop réalistes que le peintre Kobayashi Eitaku avait faites au début de l'époque Meiji (1868-1912), est-ce parce que la nudité représentait un tabou pour nos démiurges?

Les paroles qu'Izanaki et Izanami échangent après leur descente sur l'île semblent montrer qu'il n'en est rien. En forçant un peu le texte selon une logique réaliste, on pourrait supposer que, puisqu'ils parlent d'unir leurs organes génitaux (la partie trop développée ou insuffisamment développée), il y a forcément un instant où ils sont nus. Or, le caractère symbolique du langage du mythe nous prévient contre cette supposition. Mais cela n'infirme en rien notre hypothèse, car la nudité devient symboliquement présente quand ils s'interrogent sur la nature de leurs corps. Il n'y a aucune retenue, aucune honte à parler de leurs anatomies respectives, et selon 
une logique performative, on peut dire que ces mots instaurent la nudité dans la scène. Après avoir décrit mutuellement la forme de leurs corps, Izanaki informe sa conjointe du rituel qu'ils doivent effectuer, c'est-à-dire la giration autour du pilier céleste. C'est au moment de cette giration que les deux dieux se regardent et s'adressent des éloges sur leur beauté, à savoir : « Oh, le bel homme! », « oh, la belle femme! ». Difficile de savoir aussi si ces expressions se réfèrent au corps nu du conjoint ou si elles se limitent au visage, mais ce qui compte c'est que les deux dieux ne se trouvent beaux qu'après s'être fait l'aveu de leurs morphologies respectives. Puis, il faut souligner que ces exclamations ne figurent point dans les instructions données par Izanaki sur le rituel à suivre, ce qui prouve que ce sont des paroles spontanées et non pas de simples formules rituelles.

En tout cas, le regard porté sur le corps du partenaire, loin d'éveiller des sentiments négatifs comme la honte, sert de déclencheur à l'attirance mutuelle, à l'union des deux dieux et par la suite à l'enfantement des divinités de la série généalogique suivante. Il n'y a aucun tabou décelable dans cette première partie de l'épisode.

\section{... à la honte}

Izanaki et Izanami ont eu un premier enfant, Hiruko (littéralement «l'enfant sangsue »), qui est né difforme. Cet échec pousse les démiurges à consulter l'assemblée des dieux célestes, qui annonce que ce dernier est dû au fait qu'Izanami avait parlé la première. Après cette tentative ratée, nos deux acteurs recommenceront la cérémonie, mais cette fois-ci en observant à la lettre les règles sur l'ordre de prononciation. Une fois la cérémonie réussie, ils commenceront à enfanter pour de bon, d'abord des îles et ensuite des divinités. Néanmoins, après avoir donné naissance à Kagutsuchi, le dieu du feu, Izanami aura son sexe brûlé et tombera morte. Izanaki, ne pouvant pas supporter la mort de son épouse, tuera son fils et partira chercher son épouse décédée au pays des morts, Yokotsukuni. Une fois arrivé à la frontière de ce pays, il s'y entretient avec son épouse, et elle lui avoue avoir déjà mangé la nourriture du pays des morts, ce qui implique pour elle l'impossibilité de retourner dans le monde des vivants. Cependant, émue par le geste de son époux, elle éprouve le désir de repartir avec lui, et lui dit qu'elle ira en parler aux dieux de Yokotsukuni. Avant de le quitter, Izanami lui interdira de la regarder pendant qu'elle s'y trouvera. Or, la longue attente poussera Izanaki à pénétrer dans le pays des morts où il retrouvera le cadavre de son épouse rongé par des vers, vers qui deviendront les divinités du tonnerre. Horrifié à la vue du corps de son épouse, Izanaki essaiera de s'enfuir, et c'est à ce moment-là qu'Izanami s'exclame « tu m'as couverte de honte! » et ordonnera aux divinités du tonnerre de persécuter son époux. Elle se joindra ellemême à la poursuite et arrivera jusqu'à la sortie du pays des morts, où son époux avait placé un énorme rocher pour bloquer le passage. Chacun d'un 
côté du rocher, ils prononceront une formule de divorce qui sera fatalement reliée à l'instauration de la mort dans le monde".

Pourquoi ce changement de l'attirance à la honte? Certainement, rongé par des vers, il est facile de comprendre que le corps d'Izanami ne soit plus attirant, mais pourquoi la vue de ce corps représente-t-elle un tabou? Étant donné que la nudité n'est pas en cause, le tabou le plus plausible serait celui de la vision du corps d'un mort, tabou qui a été considéré par certains comme étant universel. Mais cette hypothèse est compromise par la recherche approfondie que Matsumura Takeo avait entreprise à ce sujet, passage cité par Yamada Naomi ${ }^{10}$. Il nous rappelle que regarder les morts était aussi un rituel pour apaiser l'âme du défunt, rituel que l'on peut trouver dans la culture Maya, dans la Chine ancienne et dans les îles Ryû-kyû du Japon.

On peut aussi postuler la thèse du tabou de regarder le corps féminin lors de l'enfantement. Les vers qui rongent le corps deviennent instantanément des divinités du tonnerre, et comme nous dit Alain Rocher, selon la logique mythique, ces divinités sont enfantées par Izanami, et en sont une hypostase ${ }^{11}$. Selon la culture japonaise ancienne, quand une femme accouchait, il fallait construire un pavillon de parturition à la frontière du village afin de protéger ce dernier des impuretés, ce qui implique déjà une notion de tabou par rapport à l'accouchement. De plus, il existe d'autres épisodes où l'on peut déceler ce tabou, comme celui où le dieu céleste Howori épouse la déesse marine Toyotamahime, qui, au moment où elle enfante, dit à son époux : « les êtres de notre royaume, quand ils accouchent, reprennent leur forme originaire. Donc, ne me regarde pas ». Elle se retire ensuite dans la hutte de parturition pour accoucher. Or, intrigué par les paroles de son épouse, Howori risque un regard dans la hutte et découvre que Toyotamahime était devenue un crocodile. Le cri d'épouvante qu'il pousse provoque la honte de Toyotamahime qui abandonne son enfant et disparaît dans la mer, en prononçant une formule de divorce qui séparera à jamais non seulement les deux conjoints, mais aussi la Terre et la Mer ${ }^{12}$.

Cet épisode garde une ressemblance structurale étonnante avec notre mythe d'origine, celui d'Izanaki et Izanami. Mais la clé du tabou ne se trouve pas tellement dans l'accouchement en soi mais dans les paroles de Toyotamahime : « nous reprenons notre forme originaire lors de l'accouchement ». Ceci nous montre que l'accouchement est juste une condition nécessaire au retour à la forme originaire, mais non pas la source du tabou, car ce dernier se trouve dans la vue de la vraie forme ou nature du partenaire. Pour citer Alain Rocher :

9. Kojiki. Nihon shisô taikei, op. cit., p. 33-39; Nihonshoki. Nihon koten bungaku zenshû, op. cit., p. 42-47.

10. Yamada Naomi, op. cit., p. 8.

11. Alain Rocher, Les Figures de l'origine dans la mythologie japonaise, Grenoble, Université Stendhal-Grenoble 3, 1988, p. 522.

12. Kojiki. Nihon shisô taikei, op. cit., p. 113-115. 
« Si la femme indisposée, parturiente ou mourante se dérobe aux regards, c'est pour préserver un secret qui concerne son être le plus intime ${ }^{13}$. »

Effectivement, Toyotamahime accouche sous la forme d'un être marin, et c'est cette vue qui repousse Howori, la découverte de l'altérité de son épouse. Ceci est en rapport avec un thème fréquent dans le folklore japonais, le irui (異類), ou mariage entre des espèces différentes. C'est l'époux en général qui, en découvrant la vraie forme de son partenaire, s'épouvante et essaie de s'enfuir, provoquant la honte du conjoint et la séparation par la suite.

Certes, dans notre épisode premier, Izanaki et Izanami sont censés appartenir à la même espèce car ils sont frère et sœur, mais après la mort d'Izanami, cette dernière absorbe rapidement la nourriture du pays des morts, ce qui scellera fatalement le transfert de nature. Mais sa nouvelle nature n'est pas tellement celle d'une hypothétique et abstraite " espèce des morts ", mais quelque chose de plus concret et plus en accord avec le caractère animal d'irui (異類). Izanami étant en train d'enfanter les divinités du tonnerre quand elle est surprise par Izanaki, ce seront ces divinités qui révèleront sa nouvelle nature. Alain Rocher, en se basant sur les études de Tsuda Sokichi, nous dit que les dieux du tonnerre étaient assimilés à des serpents dans le Japon ancien ${ }^{14}$. Ce sera donc la vue de cette nouvelle nature ophidienne de son épouse qui horrifiera Izanaki et qui mènera directement à la fin tragique de notre épisode. Une des versions du Nihon-shoki modifie l'exclamation de la déesse, en lui faisant dire : « tu as vu ma forme, il faut que je vois la tienne! » Ce sera cette exclamation qui effraiera Izanaki, effroi qui trahit d'une part la peur de l'altérité et d'autre part la peur d'être entraîné à son tour dans l'inconnu. Donc, si la vue de la ressemblance corporelle, ou en d'autres mots, de la parenté, entraîne l'attirance et le charme, la vue de l'altérité corporelle entraîne peur et répulsion, réaction qui provoquera à son tour la honte chez le partenaire.

Comme preuve de cette dernière affirmation, on peut mentionner au passage que pendant qu'Izanaki et Izanami sont frère et sœur, et donc parents, l'accouchement ne révèle pas une forme différente pour la femme. Effectivement, le mythe de la création nous apprend qu'Izanami ne se retire pas pour accoucher de la première série des divinités. Izanaki est donc présent, car le texte nous dit que ce sont eux deux qui donnent naissance aux divinités. Certes, l'acte de procréation et la naissance sont presque simultanés dans le langage mythique de notre épisode, et il est donc difficile de savoir si les mots « ils donnèrent naissance » font référence à l'acte de procréation ou à l'accouchement. Mais tout ce que l'on peut affirmer est qu'Izanaki ne sort pas de la scène car, quand Izanami a son sexe brûlé après avoir donné naissance à Kagutsuchi, le dieu du feu, et qu'elle meurt par la suite, le texte nous dit que dans cette même scène Izanaki verse des larmes 
pour son épouse. Néanmoins l'insistance du texte sur la participation de deux acteurs dans la naissance des divinités nous donne à penser qu'il est légitime de postuler qu'Izanaki n'est pas un simple spectateur passif et qu'il joue un rôle quelconque dans l'enfantement. Peut-être est-il en train d'assister son épouse comme une sorte de sage-femme, son rôle étant en revanche effacé par la description sérielle des îles ou des divinités enfantées.

Pour conclure cet épisode intitulé « de l'attirance à la honte », il est possible d'affirmer que dans ces textes, et pour le cas des conjoints, le regard posé sur un corps parent a le pouvoir de déclencher l'attraction et l'union, et par la suite celui de donner la vie. En revanche, le regard posé sur un corps d'une espèce différente a le pouvoir d'éveiller la répulsion, la honte et la mort, donc celui d'ôter la vie.

\section{Le pouvoir du corps nu exposé au regard}

\section{Ame no Uzume et la grotte d'Iwato}

Si dans le Kojiki et le Nihon-shoki, le thème de la dissimulation du corps à la vue d'autrui est fréquent, il n'empêche que l'on peut y trouver également le motif tout à fait opposé, celui de la monstration du corps nu. L'exemple le plus représentatif est celui de la déesse Ame no Uzume lors du rituel que les huit myriades de dieux exécutent devant la grotte d'Iwato. On se souvient que la déesse du soleil, Amaterasu, excédée par le comportement outrageux de son frère Susanowo, se cache dans cette grotte, ce qui entraînera l'obscurité totale de la Haute Plaine Céleste, et l'apparition d'innombrables divinités maléfiques. L'assemblée des dieux célestes décide donc d'exécuter un rituel pour faire sortir Amaterasu de sa grotte. Pendant la dernière partie de ce rituel, la déesse Ame no Uzume commence à danser nue devant la grotte, sera possédée par un esprit, entrera en transe et laissera tomber sa tunique en montrant ses seins et son sexe, ce qui provoquera le rire des huit myriades de dieux. Amaterasu, curieuse d'entendre des rires alors que tout aurait dû être obscur et triste du fait de son absence, entrouvre petit à petit la porte de la grotte pour regarder et sera finalement entraînée au dehors par une autre divinité. La lumière apparaîtra à nouveau dans le monde, les divinités maléfiques disparaîtront et l'ordre sera restauré ${ }^{15}$.

Ce qu'on peut voir ici, c'est que la vue du corps nu, loin d'entraîner des calamités, apporte à nouveau la restauration de l'ordre dans le monde. Le Nihon-shoki ne mentionne pas la nudité d'Ame no Uzume, et dit que c'est seulement la danse de la déesse et l'incantation qu'elle exécute qui pousseront Amaterasu à sortir de sa grotte. Ici on ne voit point de rapport entre « corps » et « regard ». En revanche, le scénario dépeint par le Kojiki insiste

15. Kojiki. Nihon shisô taikei, op. cit., p. 49-54; Nihonshoki. Nihon koten bungaku zenshû, op. cit., p. 75-78. 
sur la nudité de la déesse, en mentionnant explicitement les seins et le sexe. Le regard des huit myriades des dieux posé sur ce corps nu provoquera leur rire et par la suite la curiosité d'Amaterasu. Toutefois, il faut souligner que la danseuse ne cherche pas intentionnellement le regard des dieux, car le Kojiki insiste sur le fait qu'Ame no Uzume est possédée (kamigakari 神懸) par une divinité. Nagafuji Yasushi ${ }^{16}$ affirme très justement que cette nudité n'est pas intentionnelle, et que cela relève plutôt d'un rituel chamanique de possession, pratique fréquente dans le Japon archaïque. De plus, si les dieux rient ce n'est pas de l'obscénité du spectacle, nous dit-il, mais il s'agit en fait d'une réaction rituelle, car ils sont emplis de respect vis-à-vis de la divinité qui a possédé Ame no Uzume. Certes, relier le rire au respect peut paraître contradictoire, mais le rire fait aussi partie des rituels chamaniques et il a un pouvoir purificateur selon Nagafuji. On voit ici le pouvoir de la nudité ritualisée, puisque dans cet épisode corps et regard sont associés rituellement pour obtenir un résultat positif.

\section{La soumission à la vue du corps nu}

Il est question ici d'une deuxième apparition d'Ame no Uzume, avant la descente solennelle de Ninigi, le fils des dieux célestes. Alors que Ninigi s'apprête à descendre du ciel pour prendre possession de la Terre, une divinité terrestre, Sarutahiko, lui bloque le passage. Les dieux célestes décideront d'envoyer notre bien connue Ame no Uzume pour soumettre l'intrus. Le Kojiki nous dit que Sarutahiko, à la vue de la déesse, se présente immédiatement comme la déité qui servira de guide à l'auguste fils des dieux célestes ${ }^{17}$, mais il ne faut pas se tromper sur cette apparente soumission spontanée. Comme nous le rappelle Alain Rocher, Sarutahiko est 1'ennemi des Tenson (les descendants des divinités célestes) ${ }^{18}$, ce qui est bien logique car il s'agit d'une divinité terrestre. Rappelons au passage que Sarutahiko est dépeint comme un ennemi effrayant dans le Nihon-shoki, avec un gros nez d'une longueur de sept mains, des yeux farouchement brillants et grands comme des miroirs de huit mains de diamètre, émettant une lumière capable d'éclaircir la Haute Plaine Céleste ${ }^{19}$. Cet affreux portrait explique qu'aucune des huit myriades de divinités de Takamanohara ne soit prête à l'affronter. Les dieux Amaterasu et Takagi confient finalement cette mission à Ame no Uzume, en lui disant : " Bien que tu sois une femme petite et délicate, personne ne peut te battre du regard. Va donc questionner cette divinité qui se tient là-bas. » Le Kojiki insiste sur ce

16. Nagafuji Yasushi, Amenouzume no sei to butô [La danse et le sexe d'Ame no Uzume], Kaishaku to kanshô, 2004, p. 25.

17. Kojiki. Nihon shisô taikei, op. cit., p. 99.

18. Alain Rocher, op. cit., p. 278.

19. Nihonshoki. Nihon koten bungaku zenshû, op. cit., p. 147-148. 
pouvoir du regard d'Ame no Uzume, et de plus une note explicative ${ }^{20}$ nous apprend le caractère maléfique (jashi - 邪視) du regard de Sarutahiko. Par conséquent, quand les deux dieux se rencontrent, ce qui est manifestement en train de se produire est un « duel de regards ». Or, Sarutahiko n'offre aucune résistance et se présente tout de suite à Ame no Uzume comme la divinité qui vient guider l'auguste fils des dieux célestes.

Alors que le Kojiki passe rapidement sur les détails de la rencontre entre les deux dieux, en nous présentant la scène comme une soumission spontanée de Sarutahiko à la vue de la déesse ${ }^{21}$, la version principale du Nihon-shoki nous donne un scénario plus intéressant, car elle insiste sur la nudité de cette dernière ${ }^{22}$. Le corps nu d'Ame no Uzume possède effectivement, comme nous l'avons déjà vu lors de l'épisode de la grotte d'Iwato, un pouvoir magique et fera à nouveau preuve de ce pouvoir lors de la rencontre avec Sarutahiko, car le Nihon-shoki nous dit que c'est sa nudité qui servira d'arme pour soumettre la divinité terrestre qui s'oppose à la descente de Ninigi. Ame no Uzume ouvre sa tunique et montre ses seins et son sexe en riant, ce qui provoque l'indignation de Sarutahiko. On peut voir dans l'expression de ce dernier une nuance moralisatrice, trait qui caractérise le Nihon-shoki, mais toujours est-il que Sarutahiko finit par se rendre et s'offre pour servir de guide au fils des divinités célestes. Tatsumi Wajiro, expert en archéologie et mythologie anciennes, affirme que le regard maléfique de Sarutahiko est chassé par la nudité d'Ame no Uzume ${ }^{23}$. Les parties intimes d'Ame no Uzume attirrent les yeux de l'intrus, et par la suite neutraliseront le pouvoir maléfique de son regard. Le corps nu féminin possède donc dans ces textes, un pouvoir purificateur, capable de chasser les mauvaises influences, notamment le mauvais œil.

Ceci nous renvoie à un rituel qui était pratiqué au Japon ancien par le clan des Sarume. Les Sarume étaient des femmes qui exécutaient des danses rituelles nues afin de chasser les mauvais esprits. Rappelons au passage qu'Ame no Uzume reçoit finalement le nom de Sarutahiko car elle l'avait soumis (la prise du nom de quelqu'un était considérée comme la preuve de la soumission du donneur), et que les descendants de cette déesse seront d'ailleurs les Sarume. On voit donc ici à nouveau la connexion du mythe avec une pratique rituelle réelle.

\section{Conclusion}

Que peut-on conclure après cet examen des rapports entre le corps et le regard? Pour le dire brièvement, la valeur accordée à la vue de la nudité

20. Kojiki. Nihon shisô taikei, op. cit., p. 99.

21. Ibid., p. 99.

22. Nihonshoki. Nihon koten bungaku zenshû, op. cit., p. 131.

23. Tatsumi Wajiro, Amenouzume no kodaigaku [Études sur l'époque ancienne appliquées à Ame no Uzume], Iwanami shoten, 1992. 
dans nos textes mythologiques dépend du contexte. L'épisode d'Izanaki et Izanami démontre que la vue du corps nu peut provoquer des sentiments tout à fait opposés selon qu'il s'agit d'un corps parent ou d'un corps appartenant à une espèce différente. Pour le cas des conjoints, c'est donc la parenté ou l'altérité qui décideront en dernière instance l'attraction ou la répulsion, au moyen du regard posé sur le corps du conjoint. Tant que l'altérité reste cachée, les deux époux peuvent vivre en harmonie, mais quand cette altérité est découverte, par exemple lors de l'accouchement, il y aura répulsion vis-à-vis du corps du conjoint et il y aura aussi honte d'avoir été vu de la part de ce dernier. Ceci entraînera la séparation à jamais des deux époux, et un certain nombre de calamités.

La vue du corps nu peut aussi se relier à d'autres réactions comme le respect ou la soumission, comme on l'a vu pour les deux épisodes de la déesse Ame no Uzume. Si le regard posé sur le corps d'un partenaire d'une espèce différente peut provoquer des calamités, le regard posé sur le corps nu (pas de relation de couple dans ce cas) lors d'un rituel a le pouvoir de corriger des calamités, en faisant par exemple réapparaître la lumière dans le monde ou en chassant les éléments qui s'opposent à l'ordre établi. Il est nécessaire de signaler aussi que ces corps nus qui ont un pouvoir magique sont toujours des corps féminins, et que les parties corporelles sur lesquelles les textes insistent ont une connotation sexuelle très marquée.

Cela prouve le caractère hétérogène de nos récits. Par conséquent, il est très difficile d'avancer des affirmations générales sur le sens du regard posé sur le corps nu, encore moins sur le sens général du corps nu dans le Japon archaïque. Le rapport que les Japonais de cette époque entretenaient avec leur corps reste obscur, car tout ce que nous avons pu tirer de cette étude n'est que l'existence au Japon archaïque de certains tabous situationnels vis-à-vis de la nudité des conjoints, et de certains rituels où la nudité féminine possède une valeur conjuratrice. Et tout cela en admettant que ce rapprochement toujours délicat entre récit mythologique et réalité ethnographique est pertinent.

Néanmoins, ce qu'on peut affirmer avec plus de fermeté, d'après les détails « crus » des descriptions corporelles qu'on y voit, est que les compilateurs du Kojiki et du Nihon-shoki n'ont pas cherché à établir une morale quelconque sur le corps, à la différence de ce que l'on peut voir dans la Bible par exemple. L'on dit souvent que ces deux ouvrages ont un caractère idéologique très marqué, et que leurs contenus avaient pour objet de justifier l'ancien gouvernement impérial du Yamato ainsi que son idéologie. Si c'est le cas, on pourrait dire alors que cette idéologie impériale ne comportait pas spécialement la notion de péché charnel ou de morale sexuelle. 